\title{
Simulação analógica de configurações eletrostáticas em uma malha de resistores $^{+*}$
}

Josebel Maia dos Santos ${ }^{1}$

Doutorando do Programa de Pós-Graduação em Ensino, Filosofia

e História das Ciências

Universidade Federal da Bahia/Universidade Estadual de Feira de Santana

Feira de Santana - BA

Clebson dos Santos Cruz ${ }^{1}$

Antonio César do Prado Rosa $\mathrm{Jr}^{1}$

Centro de Ciências Exatas e das Tecnologias

Universidade Federal do Oeste da Bahia

Barreiras - BA

José Carlos Oliveira de Jesus ${ }^{1}$

Álvaro Santos Alves ${ }^{1}$

Departamento de Física - Universidade Estadual de Feira de Santana

Feira de Santana - BA

\section{Resumo}

O estudo de superfícies equipotenciais é experimento comum nos laboratórios didáticos dos cursos de graduação em Física, funcionando como um elemento articulador do processo de ensino e aprendizagem de conceitos fundamentais do eletromagnetismo clássico, tais como o campo e o potencial. No entanto, seu método tradicional de execução apresenta algumas dificuldades técnicas que tornam essa prática experimental desestimulante para os estudantes, desfavorecendo a aprendizagem significativa destes conceitos. Neste contexto, propomos uma releitura deste experimento, na qual é utilizada uma malha de resistores para a simulação analógica de um meio eletricamente homogêneo. Os resultados experimentais obtidos são plenamente satisfatórios quanto à simulação de

\footnotetext{
${ }^{+}$Analogical simulation of electrostatic configurations in a resistor lattice

* Recebido: outubro de 2018. Aceito: abril de 2019.

${ }^{1}$ E-mails: josebiao@gmail.com; clebson.cruz@ufob.edu.br; antoniocprj@ufob.edu.br; aprendizfaced@gmail.com; asa@uefs.br
} 
configurações eletrostáticas básicas, tais como cargas puntiformes ou placas planas paralelas, permitindo, inclusive, explorar temas que não são abordados no experimento tradicional, como a configuração de para-raios.

Palavras-chave: Superfícies Equipotenciais; Malha de Resistores; Simulação Analógica; Para-Raios.

\begin{abstract}
The study of equipotential surfaces is a common experiment in the undergraduate Physics courses, it acts as an articulating element of the teaching and learning process of fundamental concepts of classical electromagnetism, such as the electric field and potential. However, its traditional execution method presents some technical difficulties that makes this experimental practice discouraging for the students, disfavoring the meaningful learning of these concepts. In this context, we propose a re-reading of this experiment, in which a resistors lattice is used as an analogical simulation of an electrically homogeneous environment. The experimental results obtained are fully satisfactory with respect to the simulation of basic electrostatic configurations, such as point charges or charged parallel flat conducting surfaces, allowing, even, to explore subjects that are not approached in the traditional experiment, like the configuration of lightning rods.
\end{abstract}

Keywords: Equipotential Surfaces; Resistors Lattice; Analogical Simulation; Lightning Rod.

\title{
I. Introdução
}

É inquestionável a importância da atividade experimental, nas suas variadas abordagens, como elemento articulador de estratégias didáticas capazes de dinamizar o processo de ensino e aprendizagem nas diferentes áreas de conhecimento da Física, tanto no âmbito do ensino básico como no ensino superior (ARAÚJO; ABIB, 2003; CARRASCOSA et al., 2006; PEREIRA; MOREIRA, 2017). Em paralelo, torna-se cada vez mais comum o uso de recursos computacionais visando práticas de ensino mais contextualizadas com uma sociedade imersa nas tecnologias de comunicação e informação (NOGUEIRA et al., 2000; ARAÚJO; VEIT, 2004; PIRES; VEIT, 2006; MOTA; REZENDE JR, 2017). O resultado disto é a quantidade expressiva de trabalhos publicados propondo aparatos e atividades experimentais para modelagem de diversos problemas físicos, aliando a inserção de novas tecnologias com o uso de 
materiais de baixo custo (ver PEREZ et al., 2018; SILVA; LIMA, 2017; TADEU et al., 2018). Com base em tal conjuntura, propõe-se neste trabalho uma releitura do experimento clássico de mapeamento de superfícies equipotenciais, comum nos laboratórios didáticos de eletromagnetismo dos cursos de graduação em Física e áreas afins.

O experimento tradicional consiste na simulação analógica de campos e potenciais eletrostáticos, utilizando correntes estacionárias fluindo em um meio eletricamente homogêneo. Em geral, para simular tal meio é utilizada uma solução eletrolítica - normalmente sulfato de cobre - disposta em uma cuba com fundo de vidro e contendo uma malha quadriculada impressa, permitindo um mapeamento bidimensional (SCHIEL, 1979; LÜDKE; GRAÇA, 2011). Uma distribuição de cargas em equilíbrio eletrostático é simulada através de superfícies condutoras, com geometria apropriada e submetidas a uma diferença de potencial fixa. A técnica mais utilizada para o mapeamento de uma superfície equipotencial é a detecção por nulo (SCHIEL, 1979), ou seja, a diferença de potencial (ou a corrente) se anula entre as pontas de prova do voltímetro (ou do amperímetro) quando estas estão localizados em posições diferentes de uma mesma linha equipotencial. A aplicação da técnica permite que, para diferentes posições de uma ponta de prova de referência na cuba, sejam mapeadas diferentes linhas equipotenciais e o procedimento é repetido até que todo espaço disponível seja explorado.

A maior dificuldade para a realização deste experimento é garantir a homogeneidade do meio, o que exige cuidados não apenas com a concentração da solução eletrolítica, mas também com a limpeza e o nivelamento da cuba. Em caso contrário, um problema recorrente é a cristalização da solução eletrolítica, o que altera localmente a configuração das linhas equipotenciais obrigando a interrupção do experimento. Além disso, a variedade de distribuições de cargas eletrostáticas que podem ser simuladas no experimento depende da quantidade de superfícies condutoras previamente disponíveis, o que pode impor restrições ao experimento. Por outro lado, se a técnica de detecção por nulo tem a vantagem de fornecer diretamente o desenho das linhas equipotenciais e, portanto, a relação com a geometria das superfícies condutoras dispostas na cuba, a mesma técnica não oferece maiores informações sobre as características quantitativas do potencial e do campo elétrico. Por fim, é comum que o tratamento de dados aplicado ao experimento se restrinja ao esboço das linhas equipotenciais em uma folha de papel milimetrado e, portanto, a qualidade dos mapas produzidos varia bastante de um estudante para outro dificultando a interpretação dos resultados experimentais.

A versão do experimento proposta neste trabalho adota uma malha de resistores idênticos para simular o meio eletricamente homogêneo, em substituição à solução eletrolítica disposta em uma cuba nivelada. As superfícies condutoras, necessárias no experimento com a cuba eletrolítica, são substituídas por curto-circuitos em pontos da malha - não necessariamente adjacentes - sendo esta uma forma bastante versátil para simular uma grande variedade de configurações eletrostáticas. Associando cada ponto da malha a um elemento de matriz, é possível registrar os dados coletados de forma ordenada, obtendo-se assim a distribuição es- 
pacial do potencial elétrico. Analisando os dados experimentais, é possível construir dois gráficos: um ilustrando a distribuição espacial do potencial elétrico e exibindo as regiões de maior e menor variação relativa; outro apresentando as curvas de nível que equivalem às linhas equipotenciais sobre o plano da malha, em uma representação bidimensional.

Os resultados experimentais são plenamente satisfatórios quanto a simulação de configurações eletrostáticas básicas, tais como cargas puntiformes ou placas planas paralelas, amplamente discutidas nos livros texto de eletromagnetismo (ver REITZ; MILFORD; CHRISTY, 1982; JACKSON, 1999). Mas também é possível explorar temas mais avançados, que dificilmente são abordados no experimento tradicional da cuba eletrolítica, a exemplo do efeito de pontas (FERREIRA, 2002), que explica qualitativamente o princípio de funcionamento de um para-raios. Este artigo está organizado da seguinte forma: a seção II apresenta os fundamentos teóricos que norteiam o experimento; na seção III, é feito um relato técnico sobre a montagem da malha de resistores e uma descrição detalhada dos procedimentos experimentais; na seção IV, são apresentados e discutidos os resultados experimentais obtidos e, por fim, é apresentada, na seção $V$, a conclusão do trabalho.

\section{Equação de Laplace e simulação analógica}

Apesar do experimento de mapeamento de linhas equipotenciais ter por objetivo o estudo de configurações eletrostáticas, ele não pode ser confundido com um problema de eletrostática. Durante a sua execução ocorre um fluxo estacionário de correntes elétricas, de modo que a densidade de carga é independente do tempo e a equação de continuidade se restringe à expressão

$$
\vec{\nabla} \cdot \vec{J}=0
$$

sendo $\vec{J}$ a densidade de corrente (REITZ; MILFORD; CHRISTY, 1982; JACKSON, 1999). A lei de Ohm implica que, para um meio homogêneo, $\vec{J}$ é diretamente proporcional ao campo elétrico. Por sua vez, mesmo não derivando de uma distribuição eletrostática de cargas, o campo $\vec{E}$ é conservativo, dado que em cada ponto da malha está associado um valor fixo de potencial. Desse modo, da equação (1) obtém-se a equação de Laplace

$$
\nabla^{2} \Phi=0
$$

sendo $\Phi$ o potencial elétrico e $\vec{E}=-\vec{\nabla} \Phi$.

A partir da equação (2) é possível realizar a simulação analógica de configurações eletrostáticas de cargas, uma vez que o potencial eletrostático $\phi_{s}$, em uma região sem cargas, também obedece à equação de Laplace. De fato, a lei de Gauss (REITZ; MILFORD; CHRISTY, 1982; JACKSON, 1999) afirma que o fluxo do campo eletrostático $\vec{E}_{s}$ através de superfícies fechadas é proporcional à carga líquida $Q_{L}$ interna à superfície. Portanto, aplicando-se o teorema da divergência à lei de Gauss e sabendo que $\vec{E}_{s}=-\vec{\nabla} \phi_{s}$, deduz-se a equação de Poisson (ARFKEN; WEBER, 2007) 


$$
\nabla^{2} \phi_{s}=-\frac{\rho\left(r^{\prime}\right)}{\varepsilon_{0}},
$$

onde $\rho\left(r^{\prime}\right)$ é a densidade volumétrica de carga, e $r^{\prime}$ uma coordenada de posição interna à distribuição. Ademais, em uma região onde não há cargas, ou seja, $\rho\left(r^{\prime}\right)=0$, a equação de Poisson corresponde exatamente à equação de Laplace. Dessa forma, aplicando as condições de contorno e regularidade adequadas, é possível realizar a simulação analógica de configurações eletrostáticas a partir de um meio eletricamente homogêneo, tal como uma malha de resistores idênticos. A única restrição é que as superficies $\Phi(x, y)$, geradas a partir de medidas de diferença de potencial na malha, correspondem a soluções da equação de Laplace em duas dimensões. O mapeamento das curvas de nível obtidas de $\Phi(x, y)$ corresponde exatamente ao mapeamento das linhas equipotenciais.

De um modo geral, linhas ou superfícies equipotenciais apresentam as mesmas propriedades de simetria que as distribuições de cargas em equilíbrio. Em particular, dada uma superfície condutora, a ausência de correntes elétricas nela, faz desta também uma superfície equipotencial (REITZ; MILFORD; CHRISTY, 1982; JACKSON, 1999).

\section{Malha de resistores e procedimentos experimentais}

Com o objetivo de simular um meio eletricamente homogêneo, construímos uma matha de resistores cujos valores de resistência elétrica se encontram na mesma faixa. A utilização de resistores se deve basicamente aos seguintes fatores: não acumulam carga, possuem baixa sensibilidade a variações de temperatura, suportam valores consideráveis de corrente e têm baixo custo.

Para a montagem da malha de resistores tomamos como base as leis de Kirchhoff aplicadas a circuitos resistivos (NUSSENZVEIG, 2001), cada ponto da malha representa um nó no circuito. Desse modo, a corrente elétrica que flui por um resistor e entra no nó é repartida em valores iguais para cada um dos demais resistores que compartilham do mesmo ponto. Por exemplo, suponha que os pontos localizados na primeira e última colunas da malha, foram curto-circuitados. Desse modo, ao longo de cada linha flui uma corrente de mesmo valor $i$, enquanto que a corrente é nula nos resistores dispostos ao longo das colunas. Logo, se a malha é composta por $m$ linhas e $n$ colunas, a resistência equivalente é dada por

$$
R_{e q}=\frac{R(n-1)}{m-1}
$$

sendo $R$ o valor da resistência de cada resistor. De modo geral, a resistência equivalente entre dois pontos arbitrários de uma malha infinita de resistores idênticos é igual a $2 R / \pi$ (TADEU et al., 2018).

Nesse contexto, o primeiro passo para a montagem da malha foi selecionar 510 resistores de $1 \mathrm{k} \Omega$ e medir as respectivas resistências. Isso foi feito com um multímetro digital da marca BK® TEST BENCH®, modelo 390. O histograma obtido, ilustrado na Fig. 1, ajusta-se 
bem à distribuição normal com média $988 \Omega$ e desvio-padrão de $6 \Omega$. Desta forma, para a composição da malha, foram selecionados resistores cujas resistências possuem valores contidos no intervalo $982 \leq \Omega R \leq 994 \Omega$.

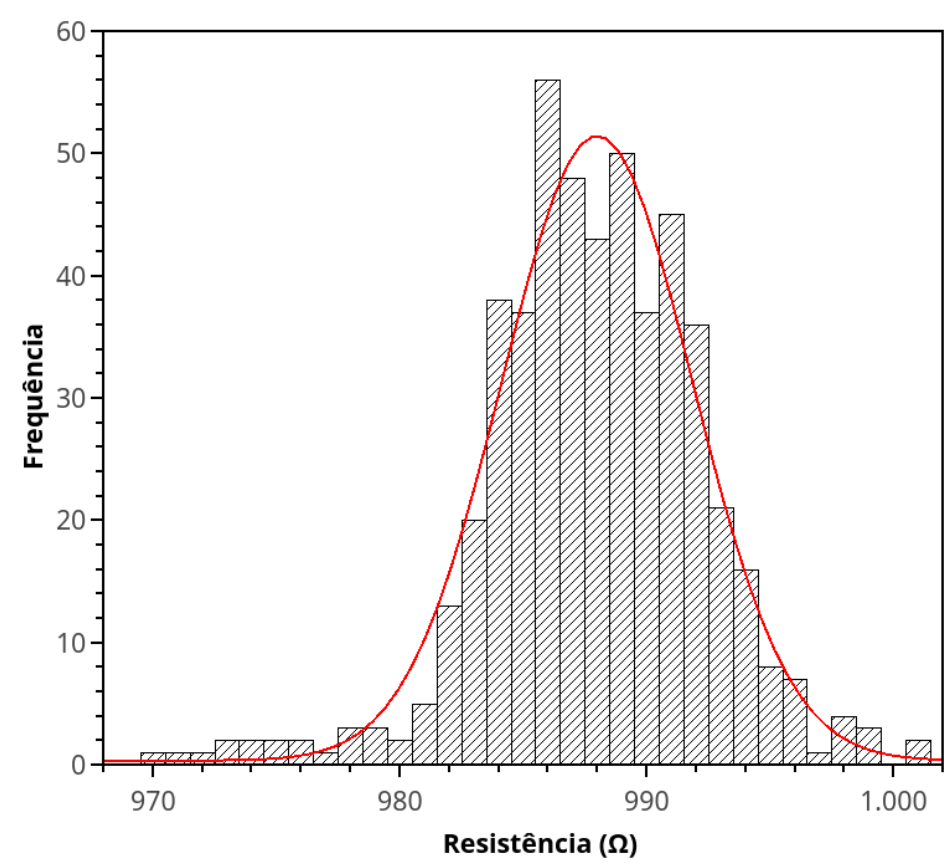

Fig. 1 - Histograma das medidas de resistência de 510 resistores de valor nominal $1 \mathrm{k} \Omega$. A linha contínua representa uma distribuição normal com média igual a $988 \Omega$ e desvio padrão $6 \Omega$.

A etapa seguinte consistiu em montar a malha em uma lâmina de acrílico com 4,0 mm de espessura. Primeiro, perfurou-se a lâmina em pontos igualmente espaçados de $1,0 \mathrm{~cm}$, com furos de 1,0 mm de diâmetro. Em seguida, os resistores foram posicionados na lâmina de acrílico e fixados a partir da torção mecânica dos terminais. Além disso, para garantir um bom contato elétrico, os terminais foram soldados. Em cada ponto interior da malha, encontram-se os terminais de 04 (quatro) resistores. Os pontos laterais reúnem os terminais de 03 (três) resistores, e os pontos dos vértices, apenas 02 (dois) terminais. Na Fig. 2, mostra-se a parte inferior e superior do protótipo, montado com 2962 resistores e que corresponde a uma "matriz de nós" de ordem $40 \times 38$, onde cada nó equivale a um elemento dessa matriz correspondente a um valor da diferença de potencial que será medido posteriormente na malha.

Após a malha ter sido construída definimos as configurações eletrostáticas que seriam simuladas, a saber: carga puntiforme positiva, cargas puntiformes simétricas, condutores planos paralelos e para-raios. Cada configuração de interesse foi então simulada através de curto-circuitos introduzidos entre diferentes nós da malha, seguindo a geometria da sua distribuição de carga. A descrição da montagem de cada configuração eletrostática está descrita na seção de resultados. Por fim, o procedimento de aquisição de dados consiste na medida, em 


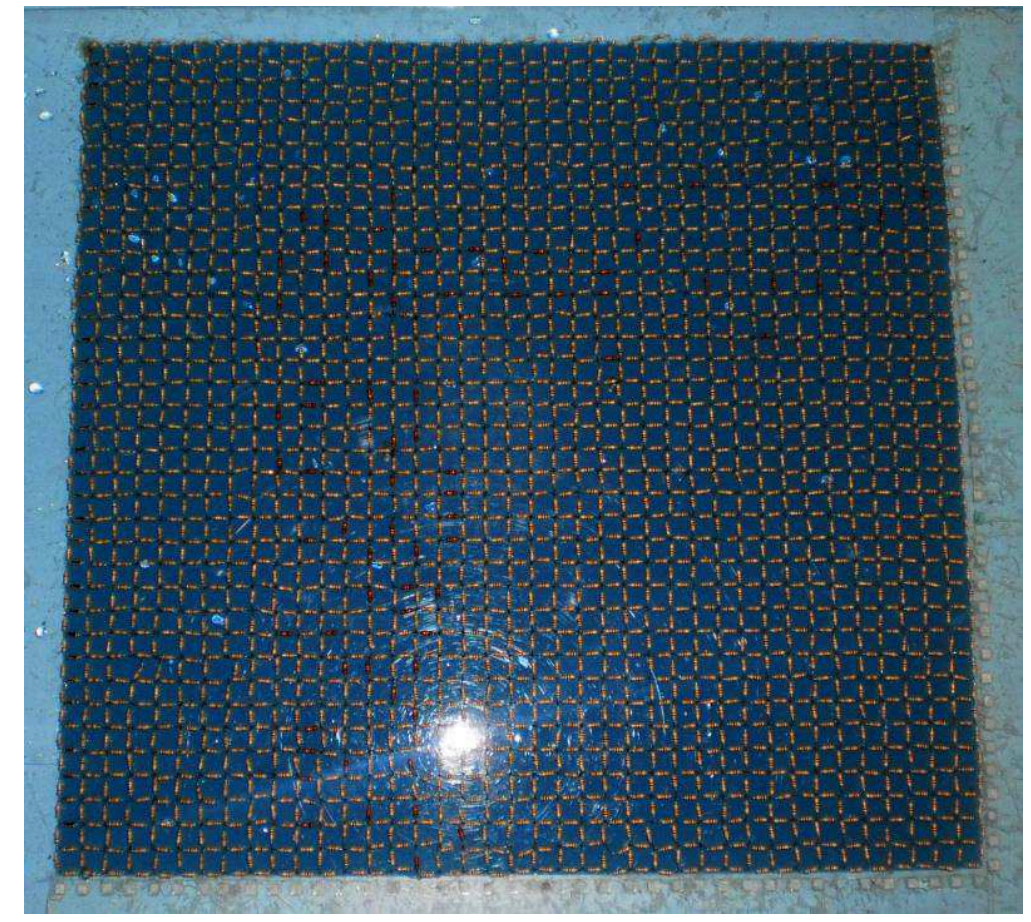

(a)

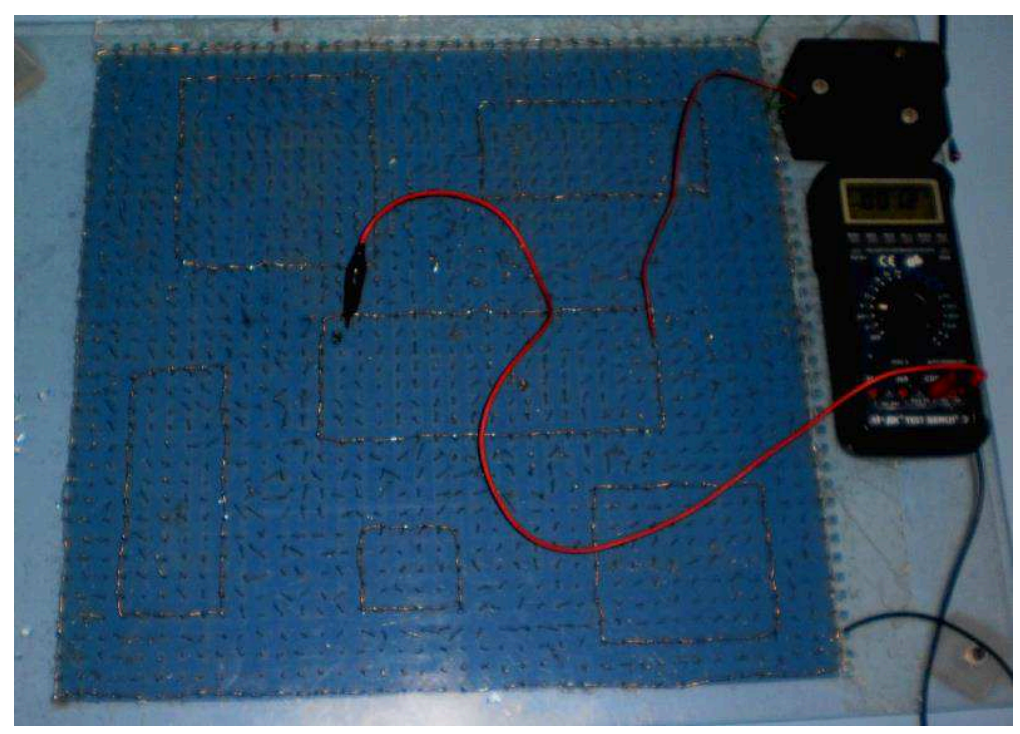

(b)

Fig. 2 - Em (a) visão da parte inferior da malha de resistores. Em (b), visão da parte superior, exibindo a rede de terminais.

cada nó, da diferença de potencial relativa a um dos curto-circuitos referentes a configuração eletrostática de interesse. Nos experimentos descritos neste artigo, foram aplicados 5,0 V utilizando uma fonte de tensão Minipa MPS-3003. O processo de medição consiste em fixar uma ponta de prova do voltímetro no terminal negativo da fonte de tensão e percorrer a malha com a outra ponta de prova, medindo assim a diferença de potencial em cada nó da malha de 
resistores. Os valores obtidos foram registrados em tabelas devidamente ordenadas, de modo que a correspondência entre a posição de cada terminal na malha e o respectivo valor de tensão fosse garantida. A partir dos dados registrados, foram determinadas as superfícies de potencial e as respectivas curvas de nível.

\section{Resultados}

Realizou-se a simulação analógica de quatro configurações eletrostáticas: carga puntiforme positiva, cargas puntiformes simétricas, condutores planos paralelos e para-raios. A seguir apresentamos a distribuição do potencial e as curvas de nível referentes às linhas equipotenciais de cada uma das configurações supracitadas.

\section{IV.1 Carga puntiforme}

A carga puntiforme foi simulada fixando-se o terminal positivo da fonte de tensão no centro da malha e aterrando-se o fio condutor conectado aos nós mais externos da rede. Desta forma, a condição de regularidade $\phi \rightarrow 0$ para $r \rightarrow \infty$ é satisfeita. Em seguida, foi estabelecida uma diferença de potencial de 5,0 volts entre os terminais, e as medições foram realizadas conforme o procedimento descrito na seção anterior. A distribuição do potencial na malha é ilustrada na Fig. 3 (a) e as curvas de nível, obtidas a partir da matriz de dados experimentais, são ilustradas na Fig. 3 (b). Observa-se a formação de curvas fechadas, concêntricas ao terminal positivo, e cujo raio médio aumenta à medida que o potencial decresce, tal como as linhas equipotenciais para uma carga puntiforme.

\section{IV.2 Cargas puntiformes simétricas}

Para a simulação de cargas puntiformes simétricas utilizou-se a fonte de tensão em configuração simétrica fixando os terminais positivo e negativo em pontos equidistantes na malha. Além disso, a borda foi aterrada. Em seguida, aplicou-se uma diferença de potencial de $-5,0 \mathrm{~V} \mathrm{a}+5,0 \mathrm{~V}$ no sistema. As Fig. 4 (a) e 4 (b) ilustram os resultados obtidos. Observa-se que as curvas de nível, obtidas a partir dos dados experimentais da distribuição espacial da diferença de potencial, são circunferências concêntricas na vizinhança imediata dos terminais, mas, à meia distância entre eles, é aproximadamente uma linha reta. 


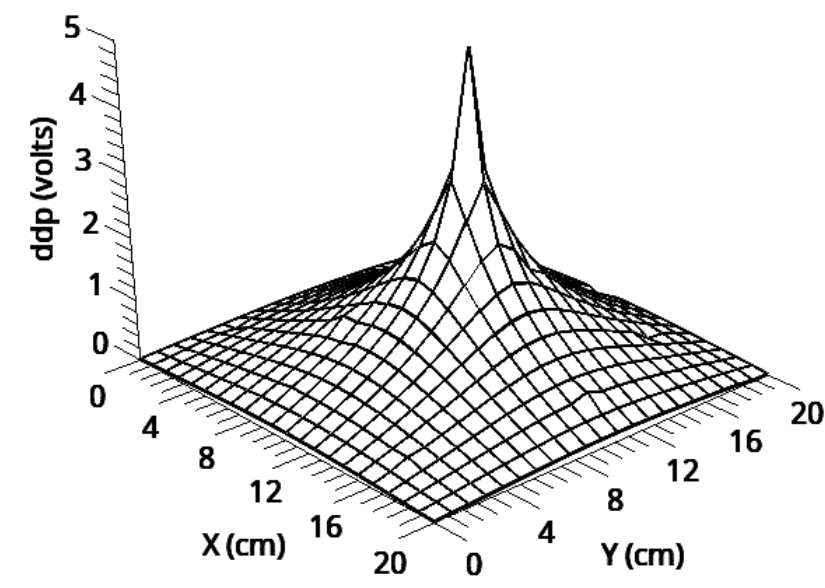

(a)

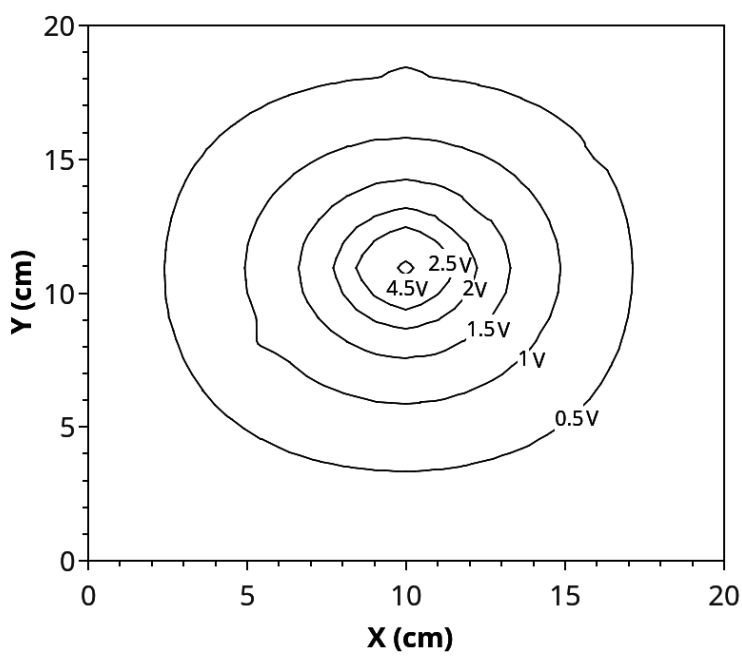

(b)

Fig. 3 - (a) Distribuição espacial da diferença de potencial (ddp) medida na malha de resistores para a simulação analógica de uma carga puntiforme na rede de resistores. (b) Curvas de nivel obtidas a partir dos dados experimentais da distribuição espacial da diferença de potencial. As curvas correspondem a círculos concêntricos centrados no ponto onde o potencial é aplicado a ddp, sendo assim uma simulação analógica de uma "carga puntiforme".

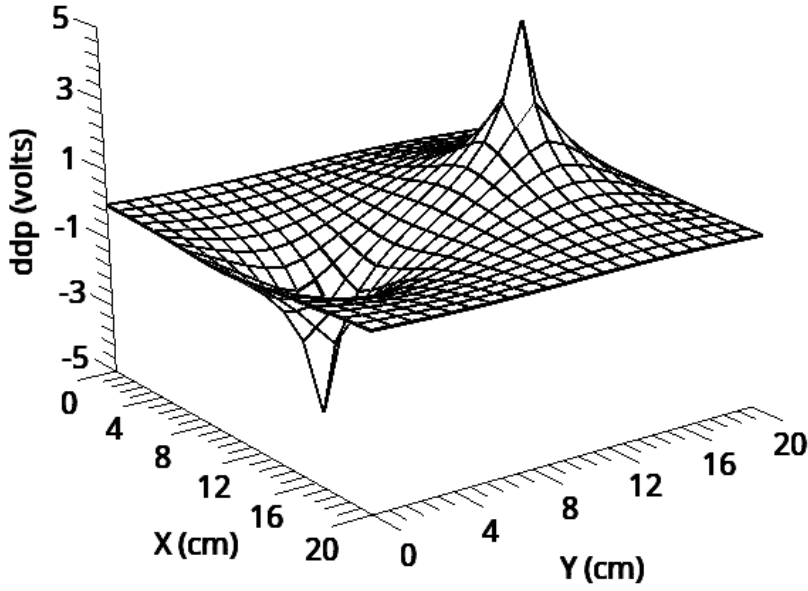

(a)

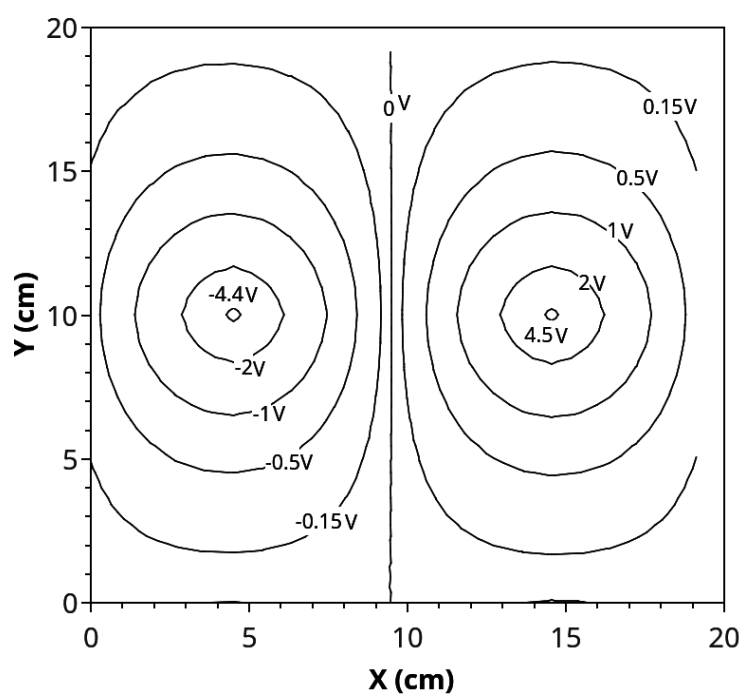

(b)

Fig. 4 - (a) Distribuição espacial da diferença de potencial medida na malha de resistores para a simulação analógica de duas cargas simétricas. (b) Curvas de nível obtidas a partir dos dados experimentais. Veem-se círculos concêntricos próximos às "cargas" e uma linha reta à meia distância entre elas. 


\section{IV.3 Placas paralelas}

A configuração de placas paralelas foi simulada através de curto-circuitos introduzidos na primeira e última linhas da malha. O terminal positivo foi fixado em um condutor e o outro foi aterrado. Os resultados obtidos são exibidos nas Fig. 5 (a) e 5 (b). Nota-se que o potencial varia linearmente entre os condutores, e as curvas de nível correspondem a retas perpendiculares ao sentido de crescimento do potencial.

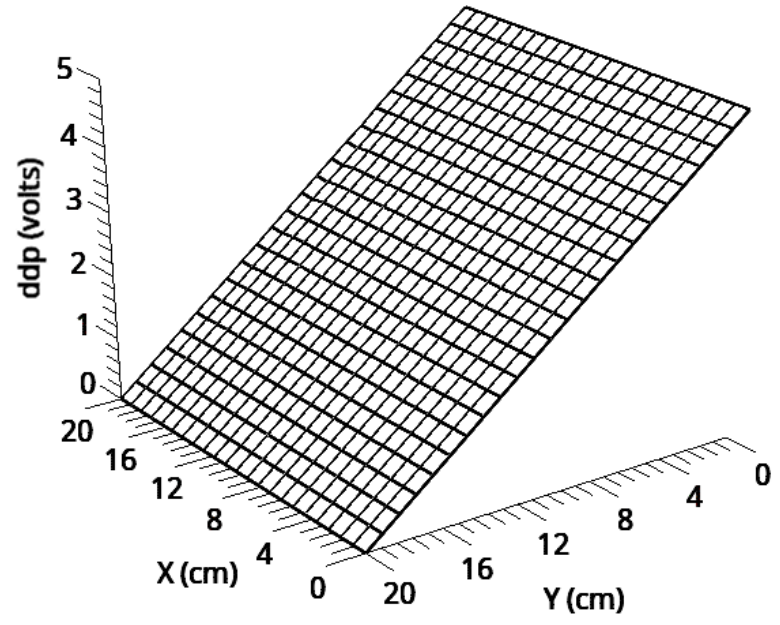

(a)

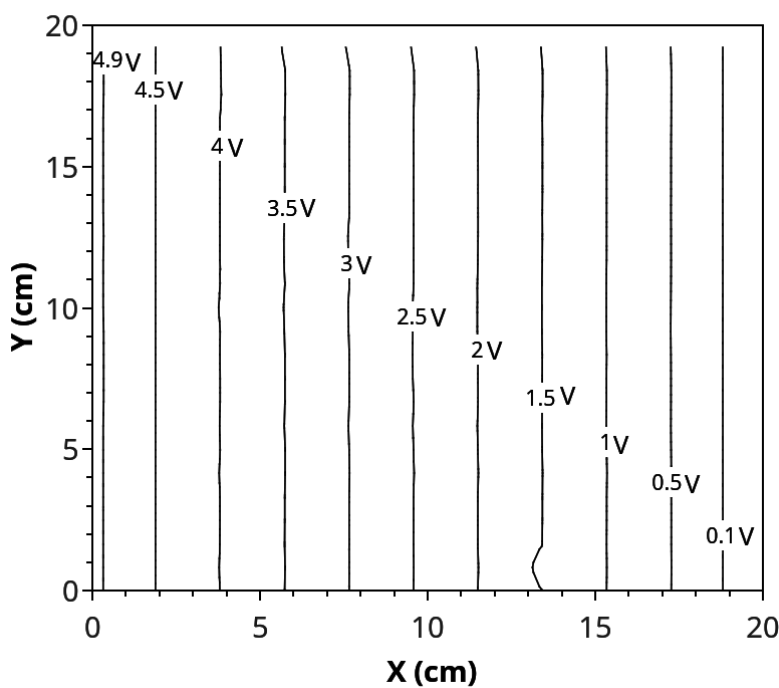

(b)

Fig. 5 - (a) Distribuição espacial do potencial para a simulação analógica de placas condutoras planas. (b) Curvas de nível obtidas a partir dos dados experimentais. Vê-se que as curvas de nivel correspondem aproximadamente a linhas retas paralelas aos condutores.

\section{IV.4 Para-raios}

A configuração da malha para a simulação do para-raios é semelhante àquela discutida para placas paralelas tendo como única diferença a inclusão de um fio condutor, fixado a uma das linhas curto-circuitadas e conectado a alguns terminais adjacentes de uma coluna mais ao centro da rede. Aterrando o conjunto, simulou-se a haste metálica de um para-raios. Por sua vez, a outra linha curto-circuitada, conectada ao terminal positivo da fonte, simula uma nuvem carregada positivamente. Os resultados obtidos são mostrados nas Fig. 6 (a) e 6 (b).

Observa-se uma mudança acentuada de curvatura e uma diminuição do espaçamento entre curvas de nível adjacentes nas proximidades da extremidade da haste. Isso implica que as linhas de campo tendem a se concentrar mais nesta região do que em qualquer outra parte do condutor aterrado, caracterizando o conhecido efeito de pontas (FERREIRA, 2002). Tam- 
bém foi simulada uma configuração com três hastes, conforme ilustram as Fig. 6 (c) e 6 (d). Percebe-se a formação de três regiões atratoras para o campo elétrico.

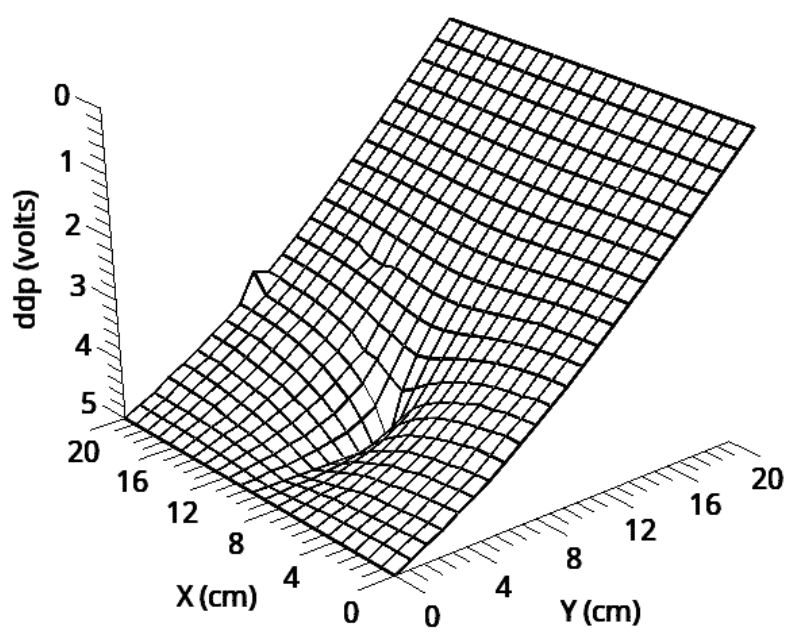

(a)

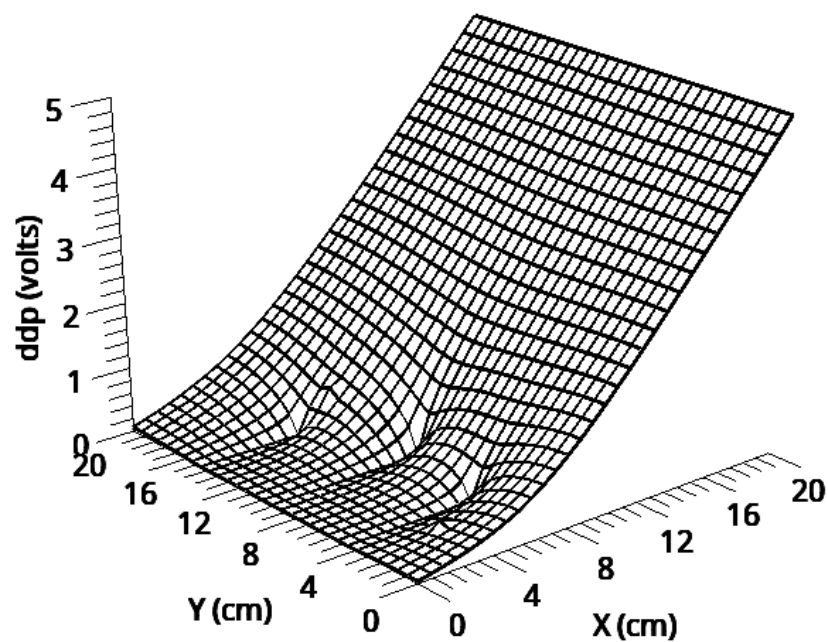

(c)

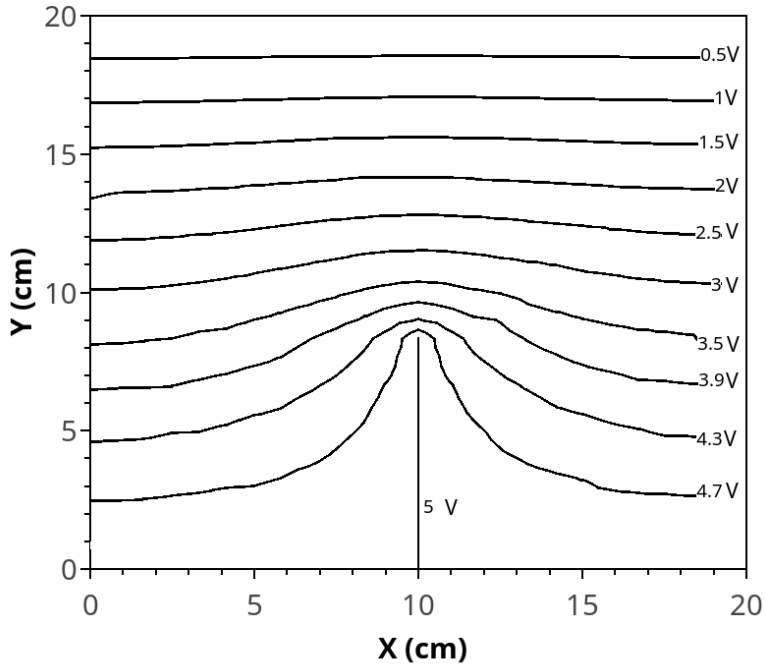

(b)

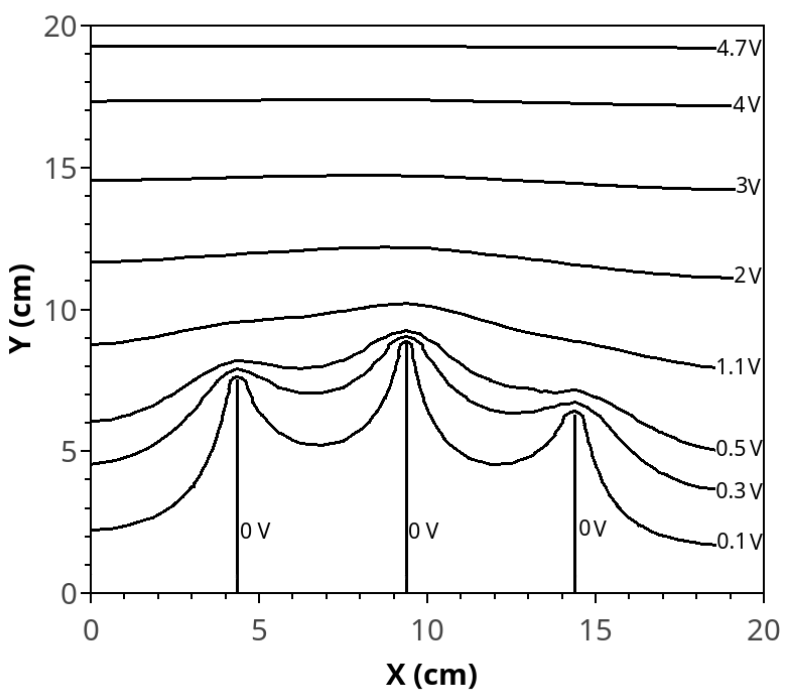

(d)

Fig. 6 - (a) Distribuição espacial do potencial para a simulação analógica do pararaios. (b) Curvas de nível dos dados experimentais para a configuração de para-raios com uma haste. As linhas equipotenciais mudam de curvatura na vizinhança da haste condutora, revelando o efeito de pontas. (c) Distribuição espacial do potencial para a simulação analógica do para-raios com três hastes condutoras. (d) Curvas de nível dos dados experimentais para a configuração de para-raios com três hastes. Permanece o efeito de pontas, mas agora existem três regiões atratoras para o campo elétrico. 
Com o intuito de compararmos qualitativamente os resultados experimentais apresentados nas Fig. 6 (b) e (d) com a solução da equação de Laplace no estudo dos para-raios, obtemos o potencial eletrostático para essas configurações implementado o método de diferenças finitas (SHADIKU, 2012). Adotadas as condições de contorno e regularidade adequadas, foi possível calcular o potencial eletrostático correspondente a cada configuração. As Fig. 7(a) e 7(b) mostram as linhas equipotenciais para o para-raios com, respectivamente, uma e três hastes. Dessa maneira mostramos que a distribuição obtida experimentalmente se assemelha à obtida através da solução da Equação de Laplace.

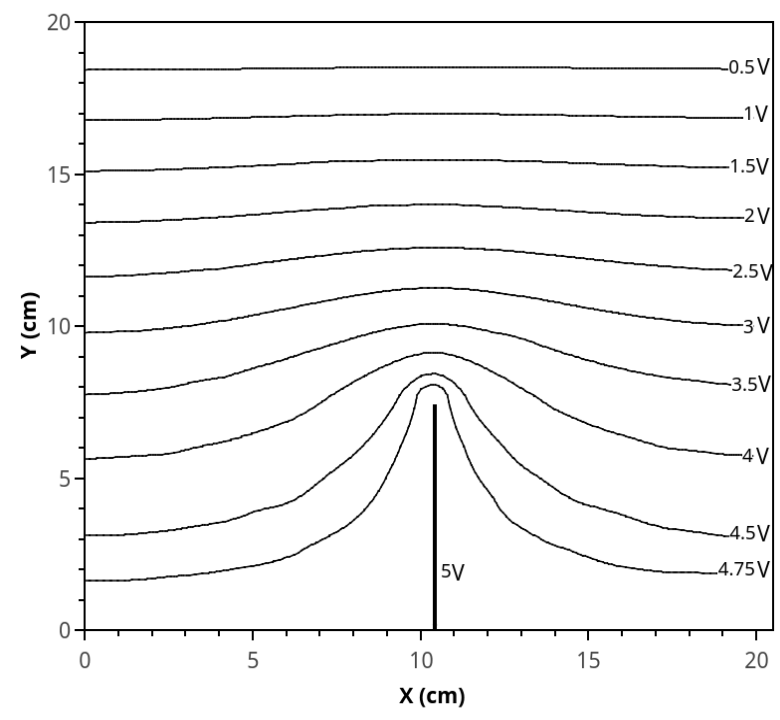

(a)

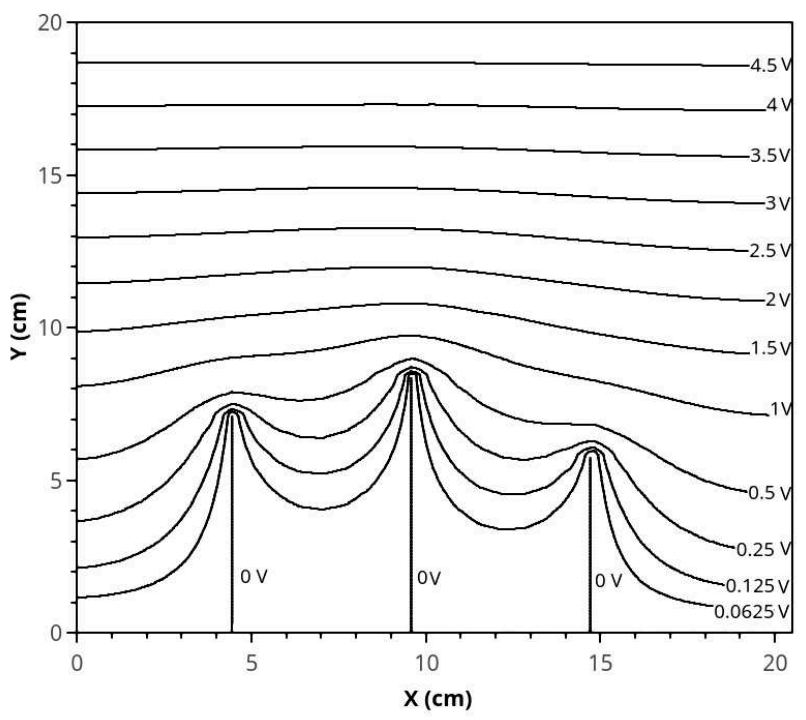

(b)

Fig.7 - (a) Distribuição espacial do potencial para a simulação analógica do pararaios com três hastes condutoras. (b) Curvas de nível da matriz de dados da solução da equação de Laplace em duas dimensões, obtida através do método das diferenças finitas. Permanece o efeito de pontas, mas agora existem três regiões atratoras para o campo elétrico, mostrando, qualitativamente, que a distribuição obtida experimentalmente (Fig. $6(b) e$ (d)) se assemelha à obtida através da solução da Equação de Laplace.

\section{Conclusão}

Os conceitos de campo e potencial são fundamentais para a devida compreensão do eletromagnetismo clássico, o que torna experimento de mapeamento de linhas equipotenciais relevante para o processo formativo dos estudantes de graduação em física e áreas afins. No entanto, as dificuldades técnicas decorrentes da utilização de uma solução eletrolítica, as limitações do método de detecção de nulo e o tratamento de dados limitado a um esboço em papel milimetrado, tornam a prática experimental enfadonha para os discentes e mascaram o seu potencial didático. 
Neste trabalho, foi proposta uma releitura deste experimento clássico, no qual o meio eletricamente homogêneo é simulado analogicamente por uma malha de resistores. Em contrapartida à técnica de detecção de nulo, a coleta de dados se dá por medidas diretas de diferença de potencial nos terminais da malha e o uso de softwares científicos permite um tratamento de dados mais eficiente.

Os resultados experimentais obtidos são satisfatórios quanto a simulação de configurações eletrostáticas básicas, tais como cargas puntiformes ou placas planas paralelas, cujas superfícies equipotenciais são amplamente discutidas na literatura. Além disso, o experimento permite explorar temas que não são abordados no experimento tradicional da cuba eletrolítica, a exemplo do efeito de pontas, que é o princípio de funcionamento de um para-raios e, no contexto adequado, pode incentivar o estudante a implementar métodos numéricos para obter soluções da equação de Laplace em duas dimensões.

Por fim, é importante salientar que se trata de um projeto de baixo custo e cujos materiais são de fácil aquisição, uma solução interessante para instituições que dispõem de poucos recursos.

\section{Agradecimentos}

Agradecemos ao apoio financeiro da FAPESB. Este trabalho é um resultado do projeto Física no Campus do Departamento de Física da Universidade Estadual de Feira de Santana (DFIS/UEFS).

\section{Referências}

ARAUJO, I.; VEIT, E. Uma revisão da literatura sobre estudos relativos a tecnologias computacionais no ensino de física. Revista Brasileira de Pesquisa em Educação em Ciências, v. 4, n. 3, 9 mar. 2011. Disponível em: <https://periodicos.ufmg.br/index.php/rbpec/article/ view/4069>.

ARAUJO, M. S. T.; ABIB, M. L. V. S. Atividades experimentais no ensino de física: diferentes enfoques, diferentes finalidades. Revista Brasileira de Ensino de Física, São Paulo, v. 25, n. 2, p. 176-194, June 2003. Disponível em: <http://www.scielo.br/scielo.php?script =sci_arttext\&pid=S1806-11172003000200007\&lng=en\&nrm=iso $>$. Accesso em 05 Apr. 2019.

ARFKEN G. B.; WEBER H. J. Física Matemática: métodos matemáticos para Engenharia e Física. Rio de Janeiro: Editora Campus, 2007.

CARRASCOSA, J. et al. Papel de la actividad experimental en la educación científica. Caderno Brasileiro de Ensino de Física, Florianópolis, v. 23, n. 2, p. 157-181, set. 2008. ISSN 
2175-7941. Disponível em: <https://periodicos.ufsc.br/index.php/fisica/article/view/6274>. Acesso em: 05 abr. 2019.

FERREIRA, G. F. L. Um Cálculo Aproximado do Poder das Pontas. Revista Brasileira de Ensino de Física, São Paulo, v. 24, n. 3, p. 367-370, Set. 2002. Disponível em: $<$ http://www.scielo.br/scielo.php?script=sci_arttext\&pid=S1806-11172002000300015\&lng $=$ en $\& n r m=$ iso $>$. Accesso em: 05 abr. 2019.

JACKSON, J. D. Classical Electrodynamics. 3. ed. Wiley, 1999.

LUDKE, E.; GRAÇA, C. O. Estudando campos elétricos de linhas trifásicas pelo método da cuba eletrolítica. Revista Brasileira de Ensino de Física, São Paulo, v. 33, n. 1, p. 01-03, mar. 2011. Disponível em: <http://www.scielo.br/scielo.php?script=sci_arttext\&pid=S1806$11172011000100027 \& \operatorname{lng}=e n \& n r m=$ iso $>$. Accesso em: 05 abr. 2019.

MOTA, A. T.; REZENDE JR, M. F. As contribuições das tecnologias da informação e comunicação em um curso de Astronomia a distância: uma análise à luz da Teoria dos Campos Conceituais. Caderno Brasileiro de Ensino de Física, Florianópolis, v. 34, n. 3, p. 971-996, dez. 2017. ISSN 2175-7941. Disponível em: <https://periodicos.ufsc.br/index.php/ fisica/article/view/2175-7941.2017v34n3p971>. Acesso em: 05 abr. 2019.

NOGUEIRA, J. S. et al. Utilização do Computador como Instrumento de Ensino: Uma Perspectiva de Aprendizagem Significativa. Revista Brasileira de Ensino de Física, v. 22, n. 4, p. 517-522, dezembro 2000. ISSN 1806 - 1117. Disponível em: <http://www.sbfisica.org.br/rbef/pdf/v22_517.pdf >. Acesso em: 05 abr. 2019.

NUSSENZVEIG, H. M. Curso de física básica: eletromagnetismo. Edgard Blucher, 2001.

PEREIRA, M. V.; MOREIRA, M. C. A. Atividades prático-experimentais no ensino de Física. Caderno Brasileiro de Ensino de Física, Florianópolis, v. 34, n. 1, p. 265-277, maio 2017. ISSN 2175-7941. Disponível em: <https://periodicos.ufsc.br/index.php/fisica/ article/view/2175-7941.2017v34n1p265>. Acesso em: 05 abr. 2019.

PEREZ, S. et al. O estudo do movimento browniano com material de baixo custo. Revista Brasileira de Ensino de Física, São Paulo, v. 40, n. 1, e1503, 2018. Disponível em: $<$ http://www.scielo.br/scielo.php?script=sci_arttext\&pid=S1806-11172018000100603\&lng $=$ en\&nrm=iso $>$. Acesso em: 05 abr. 2019.

PIRES, M. A.; VEIT, E. A. Tecnologias de Informação e Comunicação para ampliar e motivar o aprendizado de Física no Ensino Médio. Revista Brasileira de Ensino de Física, São Paulo, v. 28, n. 2, p. 241-248, jun. 2006. Disponível em: <http://www.scielo.br/scielo. php?script=sci_arttext\&pij=S1806-11172006000200015\&lng=en\&nrm=iso $>$. Acesso em: 05 abr. 2019. 
REITZ, J. R.; MILFORD, F. J.; CHRISTY, R.W. Fundamentos da Teoria Eletromagnética. São Paulo: Editora Campus, 1982.

SCHIEL D. Mapeamento de campos eletrostáticos em uma cuba eletrolítica. Revista Brasileira de Ensino de Física, v. 1, n. 1, p. 1703-1705, 1979. Disponível em: $<$ http://www.sbfisica.org.br/rbef/pdf/vol01a02.pdf>. Acesso em: 05 abr. 2019.

SHADIKU, M. N. O. Elementos de Eletromagnetismo. 5. ed. São Paulo: Bookman, 2012.

SILVA, E. S.; LIMA, A. R. Estudo da vazão de uma fonte por meio da videoanálise: uma proposta utilizando recipientes na forma de prismas regulares. Caderno Brasileiro de Ensino de Física, Florianópolis, v. 34, n. 3, p. 903-911, dez. 2017. ISSN 2175-7941. Disponível em: $<$ https://periodicos.ufsc.br/index.php/fisica/article/view/2175-7941. 2017v34n3p903>. Acesso em: 05 abr. 2019.

TADEU, E. V. C. et al. Determinação do número pi $(\pi)$ por meio de uma rede quadrada de resistores idênticos. Revista Brasileira de Ensino de Física, São Paulo, v. 40, n. 2, e2304, 2018. Disponível em: <http://www.scielo.br/scielo.php?script=sci_arttext\&pid=S180611172018000200404 \&lng=en\&nrm=iso >. Acesso em: 05 abr. 2019.

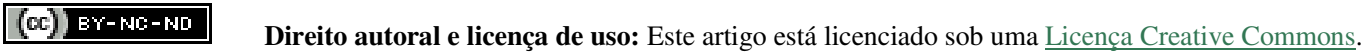

